\title{
A New Efficient and Stable 3D Conformal FDTD
}

\author{
Miguel R. Cabello, Luis D. Angulo, J. Alvarez, Member, IEEE, A. Rubio Bretones, Senior Member, IEEE, \\ Guadalupe G. Gutierrez, and Salvador G. Garcia, Senior Member, IEEE
}

\begin{abstract}
A novel conformal technique for the FDTD method, here referred to as Conformal Relaxed Dey-Mittra method, is proposed and assessed in this letter. This technique helps avoid local time-step restrictions caused by irregular cells, thereby improving the global stability criterion of the original Dey-Mittra method. The approach retains a second-order spatial convergence. A numerical experiment based on the NASA almond has been chosen to show the improvement in accuracy and computational performance of the proposed method.
\end{abstract}

Index Terms-Conformal techniques, finite-difference time domain, radar cross section.

\section{INTRODUCTION}

A major drawback of the Finite Difference Time Domain (FDTD) method [1] is its inherent staircasing approximation to structures not aligned with the Cartesian mesh, which reduces the spatial error convergence, in smooth solutions of the electromagnetic fields, from second to first order [2]. To mitigate this effect, several alternatives have been proposed, among the most popular ones being those based on geometrically conformal approaches. Some of these make use of nonorthogonal coordinate systems or fully unstructured grids [3]. Simpler approaches are based on integral forms of Ampere and Faraday laws, and give rise to the well-known Contour Path FDTD (CPFDTD) methods [4], [5]. CPFDTD has become popular for their accuracy, though the first formulations suffer from late-time instabilities which limit their applicability [6].

A modification of classical CPFDTD appeared in [7] (Dey-Mittra (DM) method) removing the late-time instability issues, and retaining its second-order spatial convergence [8]. However, the global time-step needs to be considerably reduced to ensure stability with respect to the usual FDTD CourantFriedrichs-Lewy (CFL) conditions, thus degrading its computational performance. Since this seminal work, much effort has been dedicated to studying new methods to overcome this timestep restriction.

Manuscript received November 27, 2015; revised February 15, 2016; accepted May 2, 2016. Date of publication July 21, 2016; date of current version August 5, 2016. The work described in this letter and the research leading to these results has received support from the Projects TEC201348414-C3-01 and TEC2015-68766-REDC (MINECO, Spain), P12-TIC-1442 (Junta de Andalucia, Spain), Alhambra-UGRFDTD (AIRBUS DS), and by the CSIRC alhambra.ugr.es supercomputing center.

M. R. Cabello, L. D. Angulo, A. R. Bretones, and S. G. Garcia are with the Department of Electromagnetism, University of Granada, 18071 Granada, Spain (e-mail: salva@ugr.es).

J. Alvarez and G. G. Gutierrez are with AIRBUS DS, 28906 Getafe, Spain.

Color versions of one or more of the figures in this letter are available online at http://ieeexplore.ieee.org.

Digital Object Identifier 10.1109/LMWC.2016.2588579
In [9] a variant of the DM method (Yu-Mittra (YM) method) was proposed to remove the time-step restriction by neglecting the differences in the areas of all irregular zones, which are taken as equal to that of the original Yee cell. This approach enables the authors to use the usual FDTD CFL stability criterion. The YM method is equivalent to introducing an artificial magnetic medium in conformal cells, which reduces its accuracy to first-order in space. This issue was overcome in [10] by employing an effective permeability for the magnetic field update and an electric permittivity for the electric field update (BenklerChavannes-Kuster (BCK) method). A trade-off between the time-step and the accuracy, through a closed-form expression, can be found for the BCK method, which converges to that of the YM method at the usual CFL stability limit. Another technique, the Enlarged Cell Technique (ECT) proposed in [11], is based on enlarging the irregular cells and intruding on their neighbors. Although this method succeeds in improving the stability constraints for all the cases presented therein, the requirement of enlarging the problematic cells is not straightforwardly applicable in complex geometries, since intruded cells cannot be intruders in turn. Moreover, the ECT method requires a larger stencil, which is a significant change in the traditional FDTD algorithm implemented in already-existing codes. In [8], a technique called Uniformly Stable Conformal (USC) is proposed in the context of the Finite Integration Technique (FIT), claimed to be geometrically equivalent to ECT for FDTD. The authors study two alternative methods consisting of modifying the area or the lengths of the irregular cells. They demonstrate an improvement in the time-step constraints without the need of enlarging the stencil. The authors also show that for certain cases they are able to achieve second-order accuracy.

In the present work a different approach is proposed, based on the relaxation of the DM mesh. This idea enables direct control of the time-step reduction compared to the FDTD CFL criterion, leading to a new method here referred to as the Conformal Relaxed Dey Mittra method (CRDM). In the rest of this letter, after describing the method and its properties, we present results of a numerical experiment supporting the claims made about its stability and showing a second-order spatial convergence.

\section{The Dey-Mittra Method}

The well-known DM method [7] is based on splitting the cells centered in the $\mathrm{H}$-field components which are intersected by material boundaries, into two zones of surfaces $A_{1}$ and $A_{2}$ (Fig. 1). Each zone now has a magnetic field $H^{1}$ and $H^{2}$ associated with it. These fields are updated by using the closed line integral of the tangential electric fields along their contour, 


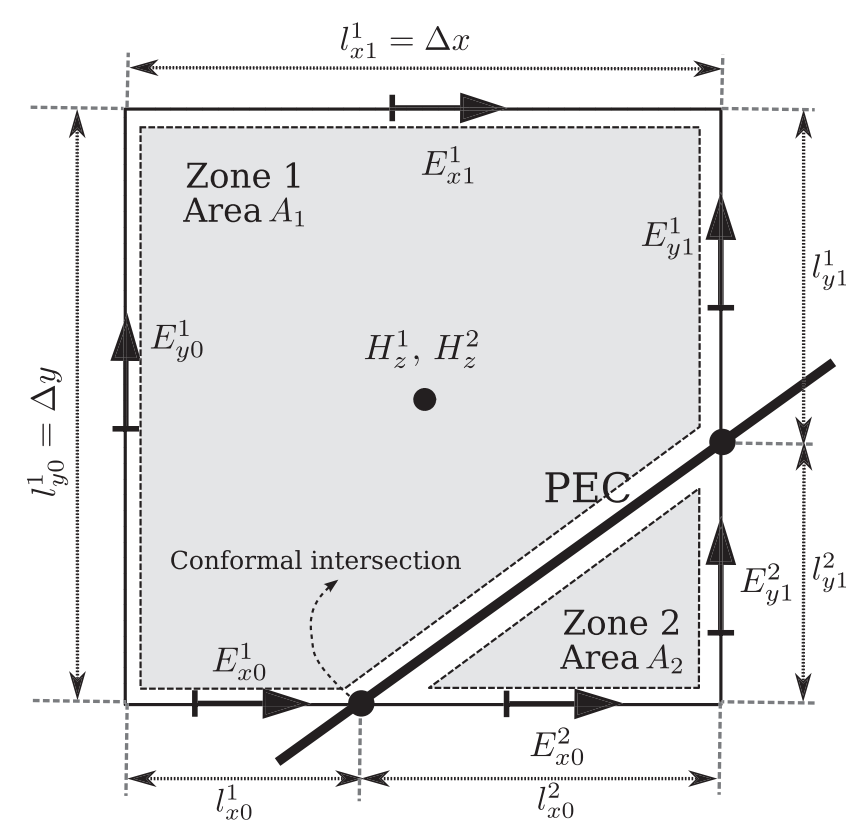

Fig. 1. Example of cross section of a conformal cell in the $x-y$ plane.The thick black line represents a PEC surface dividing the cell into two zones.

according to Faraday's law integral. For instance, in the case of Fig. 1, the magnetic fields are updated as

$$
\begin{gathered}
H_{z}^{1, n+1}=H_{z}^{1, n}-\frac{\Delta t}{\mu_{0} A_{1}}\left(l_{y 1}^{1} E_{y 1}^{1, n+1 / 2}+l_{x 0}^{1} E_{x 0}^{1, n+1 / 2}\right. \\
\left.-l_{x 1}^{1} E_{x 1}^{1, n+1 / 2}-l_{y 0}^{1} E_{y 0}^{1, n+1 / 2}\right) \\
H_{z}^{2, n+1}=H_{z}^{2, n}-\frac{\Delta t}{\mu_{0} A_{2}}\left(l_{y 1}^{2} E_{y 1}^{2, n+1 / 2}+l_{x 0}^{2} E_{x 0}^{2, n+1 / 2}\right)
\end{gathered}
$$

while the E-fields are updated by the usual FDTD algorithm.

The main drawback of this scheme is that it requires a more restrictive time-step stability condition than that imposed by the FDTD CFL criterion. We find that the DM stability condition has the same behavior of that reported in [10], except for a constant heuristical factor $\sqrt{3}$, numerically found

$$
\begin{aligned}
& \qquad t_{k}=\sqrt{3} \sqrt{\frac{A_{k} \Delta}{A_{\mathrm{FDTD}} \max \left(l^{k}\right)}} \Delta t_{\mathrm{CFL}}=h_{0, k} \Delta t_{\mathrm{CFL}} \\
& \text { with } h_{0, k}=\sqrt{3} \sqrt{A_{k}^{\text {ratio }} / \max \left(l^{k, \text { ratio }}\right)} \leq 1.0
\end{aligned}
$$

where $k=1,2$ and $A_{\mathrm{FDTD}}, \Delta t_{\mathrm{CFL}}$ are respectively, the area and time step in the CFL limit for the usual Yee algorithm, $l^{k}$ are the lengths of the usual discrete E-Field components for the zone $k$.

\section{NEW AlgORITHM: CRDM}

In this letter, we present a new strategy to fix a value for $h_{0}$ in (4), and then automatically modify the mesh to allow only certain zones of the intersections over the edges (Fig. 2). For this aim, we shift the point of the original conformal intersection, $l_{c}$, to the closest point outside a region delimited by a forbidden length $l_{f}$, whose range is between 0 and $\Delta / 2$. The new intersection will have a location defined by a relaxed length $l_{r}$

$$
l_{r}= \begin{cases}0.0 & \text { if } \quad l_{c} \leq \frac{l_{f}}{2} \\ l_{f} & \text { if } \quad l_{f}>l_{c}>\frac{l_{f}}{2} \\ l_{c} & \text { if } \quad l_{c}>l_{f} .\end{cases}
$$
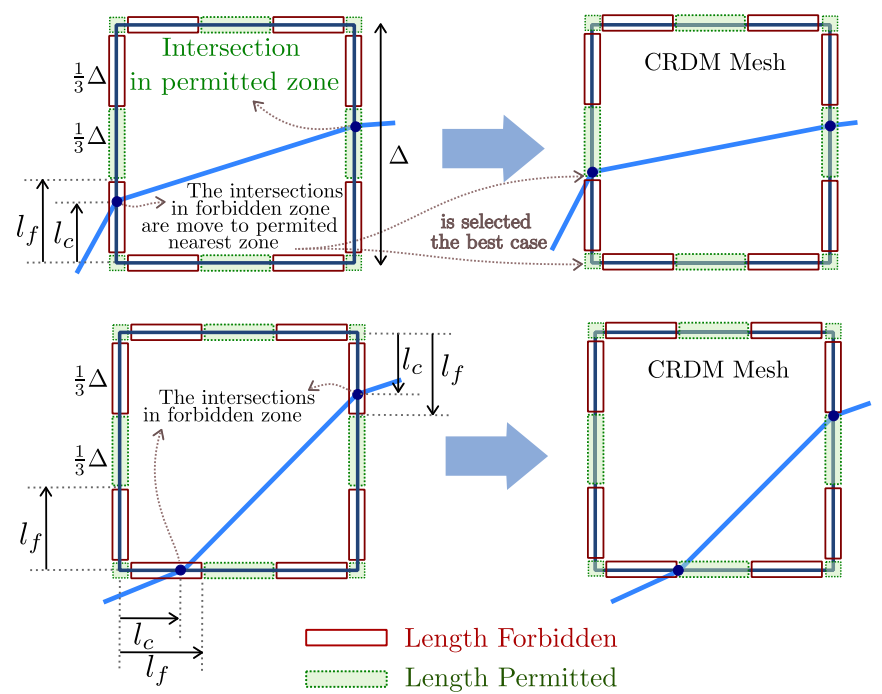

Fig. 2. Examples of the CRDM relaxation for $F_{\mathrm{rlx}}=1 / 3$ in which the geometry (dashed line) intersects the left and right edges that will be used for the simulation, represented by a simplified surface (continuous line).

Although we present the method as a mesh relaxation, it can also be regarded as a geometrical interpretation of a numerical scheme formulated as a modification of the coefficients in (1) and (2). If we define the relaxation factor $F_{\mathrm{rlx}}=l_{f} / \Delta$ as the relative distance over the edge of the Yee's cell which determines where the conformal intersections will be located, the stability criteria (3) now becomes

$$
\Delta t=\min _{\text {Allcells }}\left(h_{0, k}\right) \Delta t_{\mathrm{CFL}}=\sqrt{3 F_{\mathrm{rlx}} / 2} \Delta t_{\mathrm{CFL}} .
$$

The coefficients in (1) and (2) are modified in a way consistent with the relaxed mesh that guarantees the coherence of the magnetic flux. Finally, the relaxed mesh automatically filters out negligible areas which, having small $l^{k}$, are responsible for the late-time instabilities in the DM method. This also improves the computational costs, reducing the duplicated degrees of freedom when $l_{r}=0.0$. Note that no artificial magnetic media are introduced, as opposed to the YM and BCK methods. Numerical experiments in Section IV will demonstrate that this technique allows us to retain the second-order convergence of the classical FDTD method.

\section{NumericAl RESUlts}

The CRDM algorithm, described above, has been implemented in the SEMBA-UGRFDTD solver [12], and has been assessed with the perfectly electrically conducting NASA almond problem, reported by some of the present authors in [13]. Its RCS has been employed to assess both the accuracy and computational performance of the proposed method. The nearto-far field transformation has been computed using the geometric mean method [14] which significantly improved the results reported in [13] of the FDTD, which was found with the usual arithmetic mean [1].

Results for monostatic RCS from 0.5 to $2 \mathrm{GHz}$, and bistatic RCS at $1 \mathrm{GHz}$, are shown in Figs. 3 and 4, respectively, showing good agreement with the MoM/DGTD results. As a figure of merit for the different methods, the error of the global $L^{2}$ norm with respect to MoM/DGTD was used (Table I), indicating a 


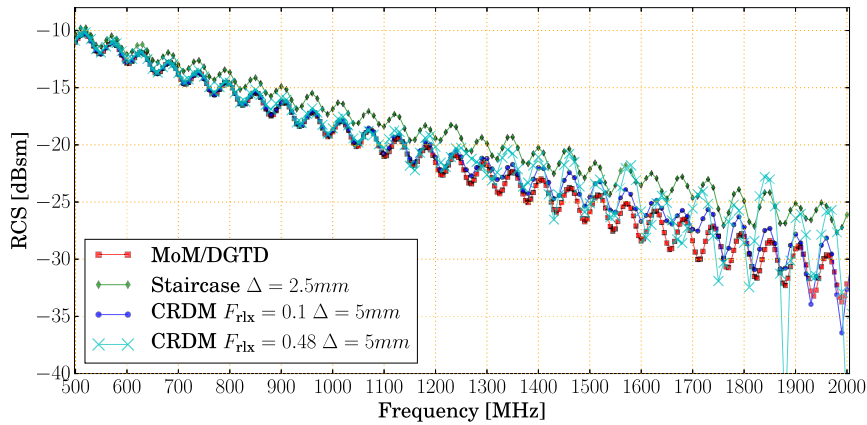

Fig. 3. Monostatic RCS of the NASA almond. Comparison resultsbetween staircase, conformal relaxed and MoM/DGTD.

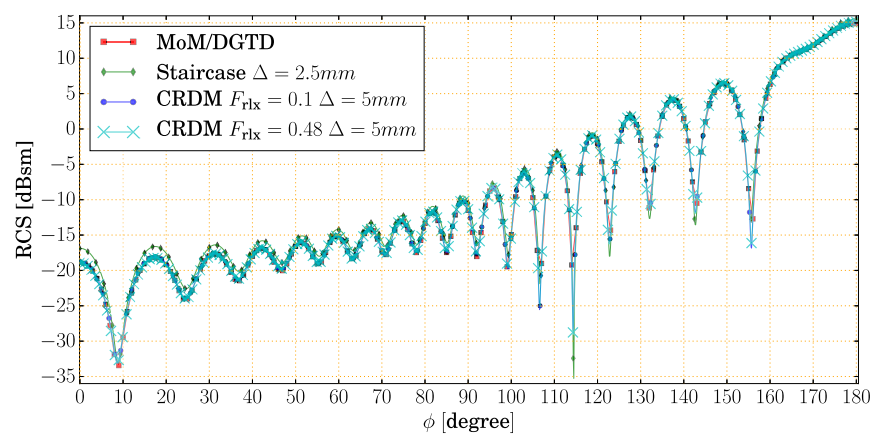

Fig. 4. Bistatic RCS of the NASA almond at $1 \mathrm{GHz}$. Comparisonresults between staircase, conformal relaxed and MoM/DGTD.

TABLE I

ERROR AS A FUNCTION OF THE CFL NUMBER FOR RCS

\begin{tabular}{lcccc}
\hline \multirow{2}{*}{ Mesh Type } & \multirow{2}{*}{$h_{0}$} & \multirow{2}{*}{ CPU[min] } & $L_{\text {bi }}^{2}\left[10^{-4}\right]$ & $L_{\text {mon }}^{2}\left[10^{-5}\right]$ \\
\hline DM 5.0mm & 0.31 & 112 & 2.13 & 2.73 \\
$F_{\text {rlx }} 0.15 .0 m m$ & 0.44 & 111 & 2.13 & 2.70 \\
$F_{\text {rlx }} 0.35 .0 \mathrm{~mm}$ & 0.71 & 74 & 2.16 & 3.63 \\
$F_{\text {rlx }} 0.485 .0 \mathrm{~mm}$ & 0.84 & 49 & 2.23 & 5.83 \\
staircase $5.0 \mathrm{~mm}$ & 0.99 & 47 & 8.22 & 22.54 \\
staircase $2.5 \mathrm{~mm}$ & 0.99 & 770 & 4.834 & 11.03 \\
\hline
\end{tabular}

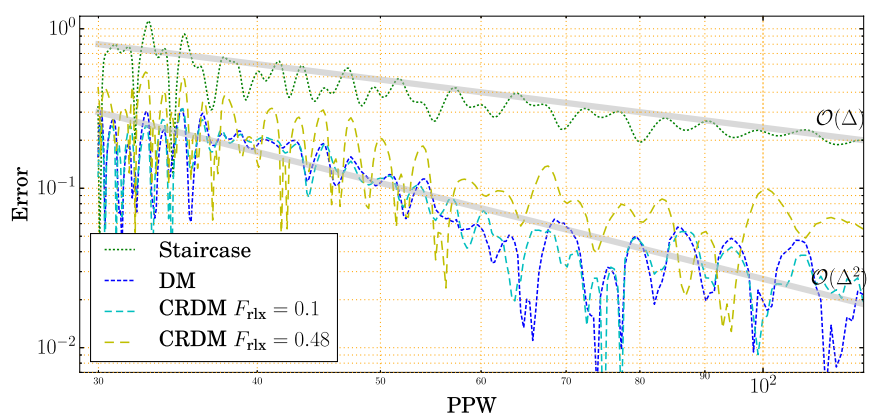

Fig. 5. $L^{2}$ error norm with respect to MoM/DGTD versusthe number of Points Per Wavelength (PPW) for the different methodsunder study.

significant improvement with respect to FDTD and with small differences among them. We also observe a significant improvement on comparing the most relaxed case with the FDTD for a doubled spatial resolution.

The $L^{2}$ error norm with respect to MoM/DGTD in a frequencies range has also been used to study the convergence of the monostatic RCS with respect to the spatial resolution. As shown in Fig. 5, the DM and CRDM methods have a convergence of
$\mathcal{O}\left(\Delta^{2}\right)$ while the FDTD has $\mathcal{O}(\Delta)$ as it is severely affected by the staircasing effect. This figure also shows that the method seems to have a similar order of convergence for all the relaxation factors studied.

For all methods using the same spatial resolution, we find the CPU time to be inversely proportional to the CFL number, $h_{0}$, implying a negligible cost on the additional operations needed in conformed cells.

\section{CONCLUSION}

A new conformal CRDM strategy has been introduced as a way of mitigating the time-step constraint of the DM method while retaining a second-order spatial convergence. The results show a significant improvement in accuracy compared to FDTD even outperforming the classical FDTD with twice the spatial resolution used by CRDM.

\section{REFERENCES}

[1] A. Taflove and S. C. Hagness, Computational Electrodynamics: The Finite-Difference Time Domain Method, 3rd ed. Norwood, MA, USA: Artech House, 2005.

[2] I. Zagorodnov, R. Schuhmann, and T. Weiland, "A uniformly stable conformal FDTD-method in Cartesian grids," Int. J. Numer. Modelling: Electron. Netw., Devices Fields, vol. 16, no. 2, pp. 127-141, 2003.

[3] R. Holland, "Pitfalls of staircase meshing," IEEE Trans. Electromagn. Compat., vol. 35, pp. 434-439, Nov. 1993.

[4] T. Jurgens and A. Taflove, "Three-dimensional contour FDTD modeling of scattering from single and multiple bodies," IEEE Trans. Antennas Propag., vol. 41, no. 12, pp. 1703-1708, 1993.

[5] S. Gonzalez, T. Materdey Bao-Hung, B. Garcia Olmedo, and R. Gomez Martin, "Volume-conformation method to study scattering by PEC objects with FDTD," Proc. Inst. Elect. Eng., vol. 143, no. 2, pp. 131-136, 1996.

[6] C. Railton and I. Craddock, "Analysis of general 3-D PEC structures using improved CPFDTD algorithm," Electron. Lett., vol. 31, no. 20, pp. 1753-1754, 1995.

[7] S. Dey and R. Mittra, "A locally conformal finite-difference time-domain (FDTD) algorithm for modeling three-dimensional perfectly conducting objects," IEEE Microw. Guided Wave Lett., vol. 7, pp. 273-275, Sep. 1997.

[8] I. Zagorodnov, R. Schuhmann, and T. Weiland, "Conformal FDTDmethods to avoid time step reduction with and without cell enlargement," J. Comput. Phys., vol. 225, no. 2, pp. 1493-1507, 2007.

[9] W. Yu and R. Mittra, "A conformal FDTD algorithm for modeling perfectly conducting objects with curve-shaped surfaces and edges," Microw. Opt. Technol. Lett., vol. 27, no. 2, pp. 136-138, 2000.

[10] S. Benkler, N. Chavannes, and N. Kuster, "A new 3-D conformal PEC FDTD scheme with user-defined geometric precision and derived stability criterion," IEEE Trans. Antennas Propag., vol. 54, pp. 1843-1849, Jun. 2006.

[11] T. Xiao and Q. H. Liu, "A 3-D enlarged cell technique (ECT) for the conformal FDTD method," IEEE Trans. Antennas Propag., vol. 56, pp. 765-773, Mar. 2008.

[12] "SEMBA, Simulador Electromagnetico de Banda Ancha." [Online]. Available: http://www.ugrfdtd.es/

[13] J. Alvarez, J. M. Alonso-Rodriguez, H. Carbajosa-Cobaleda, M. R. Cabello, L. D. Angulo, R. Gomez-Martin, and S. G. Garcia, "DGTD for a class of low-observable targets: A comparison with mom and $(2,2)$ FDTD," IEEE Antennas Wireless Propag. Lett., vol. 13, pp. 241-244, 2014.

[14] J. B. Schneider, Understanding the Finite-Difference Time-Domain Method, 2010. [Online]. Available: http://www.eecs.wsu.edu/schneidj/ uFDTD/ 